\title{
THE CONSTRUCTION TECHNOLOGY OF THE BOUNDARY ENVIRONMENT MODEL BASED ON HEXAGON
}

\author{
Lili Wu $^{\mathrm{a}, *}$, Yixin Hua ${ }^{\mathrm{a}}$, Qing Xu ${ }^{\mathrm{a}}$, Yang Cheng ${ }^{\mathrm{b}}$ \\ a PLA Information Engineering University, Zhengzhou ,China- wll_sx_1984@163.com \\ b School of Geography, Beijing Normal University, Beijng, China- chengyangAbnu.edu.cn
}

KEY WORDS: Spatial, Structure, Technology, Terrestrial, Thematic

\begin{abstract}
:
Since ancient times, land boundary problem is one of the most sensitive issues. The study of land boundary environment, is the foundation of safeguarding state sovereignty and territorial integrity. The land boundary environment includes natural geographical environment, human geographical environment and other aspects. These complicated information gathering at the land border area, to a certain extent, affects the land border demarcation and analysis. Although there are a number of ways to organize, manage and express these information, but these methods lack the entire description and abstract to the whole land boundary environment, and the correlation between the information is not strong, the most important is that they can not make the continuous border environment information participate in the process of analysis and demarcation of boundary. Therefore, this paper analyzed the specific content of the land boundary environment and its shortcomings in the land delimitation, proposed the Hexagonal Land Boundary Environment Model both with the hierarchy and integrity, expounded the superiority of the model, and discussed the process and methods of how to build the model.
\end{abstract}

\section{THE LAND BORDER ENVIRONMENT OVERVIEW}

\subsection{The Content of The Land Boundary Environment}

Land boundary conditions are complicated, the different land border areas contain different information contents. But overall, it mainly includes the following several aspects[1][2][5]:

\section{(1) Natural geographical factors}

Natural geographical factors mainly include all kinds of landform, climate, hydrology, land ocean partition, soil type, etc. It is the foundation of the people relies for survival and development, is also the main stage of nation and state fight for.

(2) Human geographical factors

Human geography factors mainly include administrative divisions, population, ethnic, cultural level, and so on. These are not only the boundary integral part of the environment, but also affect the boundary area is final ownership.

\section{(3) Social and economic factors}

Social and economic factors mainly include land resources, water resources, mineral resources, forest resources, animal and plant resources, and so on. Today, much attention has been paid to energy and mineral resources, the basis status of social economic elements especially the resources and the protection function to the national security are more and more attention by every government[8].

(4) Historical and political factors

Historical and political factors mainly include left over by history, an integral and other factors. The impact of the history on the border area is long. it not only affects the scope of countries and regions of the earth, also affect the country and border area of politics, economy, culture, ethnic groups, and even of the distribution and scope of the religion[7].

\subsection{The Land Border Environment Analysis}

The way of the existing land boundary information participate in the demarcation and the analysis is limited, the main disadvantages are as follows:

(1) The process that the demarcation information participate in decision-making is completed in the brain, that is bad for the summary of the information to participate in the way, but also not conducive to the transfer of demarcation experience;

(2) The demarcation information cannot be involved in the demarcation process, which makes the demarcation process has certain blindness;

(3) The continuity of demarcation information makes it unable to realize computer aided generation of demarcation scheme. Decision makers can only artificially demarcate. The workload of modify is larger.

Through the analysis of the land boundary conditions, the insufficiency of its in decision analysis, the author first puts forward the Hexagon-Based Land Boundary Surroundings Model.

\section{CONSTURCTION OF THE HEXAGON-BASED LAND BOUNDARY SURROUNDING MODEL}

\subsection{The Concept of Model}

The Hexagon-based Land Boundary Surroundings Model(HBLBSM),is secondary modeling and organization to the existing land boundary conditions through the hexagonal grid subdivision to the land border area. It make the vital information of area mapped to each hexagonal lattice and provide related analysis capabilities to achieve the purpose of land border environmental information discretization and laying 


\section{5 - 6 December 2013, Beijing, China}

the groundwork for further decision and evaluation, especially the generating of initial solution line[3].

\subsection{The Choice of Hexagon}

Regional subdivision has many choices, such as triangle, square and hexagon. Compared with other two kinds of graphics, hexagon is slightly complicated, but it has the following features[3][4]:

(1) The hexagon coverage rate is wider. Compared with several other graphics, in the case of equal length, hexagon is the largest and the coverage is also the widest.

(2)The hexagon is the same proximity. There are six edge adjacent cells of hexagon, and no angle of adjacent cell, and the distance that the polygon center to the center of the adjacent units are always equal.

(3)There are only connected edges between adjacent hexagon units. This makes the image won't disrupt like other graphics, and the tend of the border in the boundary conditions will be more smooth and continuous.

(4) The hexagon has cells

There are only hexagon cells that the sides nearby, no hexagon cells that the angle nearby. This will not increase the complexity of the adjacent analysis;

(5)The hexagon is the most compact. Compared with the triangle and square, in the condition of the equivalent resolution, the sampling density of the hexagon is the largest, the resolution is the highest, and the land boundary environmental expression is more precise and detailed.

(6) The hexagon has better angular resolution. The angular resolution of the triangle is 120 degrees, the square resolution of the triangle is 90 degrees, and the hexagon resolution of the triangle is 60 degrees. It is helpful to improve the accuracy of the pattern recognition, the road extraction and feature line extraction.

(7) Compared with the square and triangle, the direction that the hexagon described is more, which makes the border more flexible, the information that the model of expressed more real.

To sum up, the model selects the hexagon as the basic graphic which makes the land boundary information discretization.

\subsection{Model Building Process}

The HBLBSM is a reconstruction of the surface of the environment information, is conceptually equivalent to the logical partition to digital map.To the information originally related and detached by hexagons, we can define topology relationship between hexagons to supplement the description. The process of the model construction is shown in the figure below:

(1) Determine the land border regions, and clear classification rules and mathematical foundation. On this basis, complete the area subdivision by the user participation.

(2) Select the information types and content, complete the information mapping in the mapping rules, construct the model contains preliminary information.
(3) For the specific information, through artificial participation way to supplement and perfect, complete the model satisfying demand.

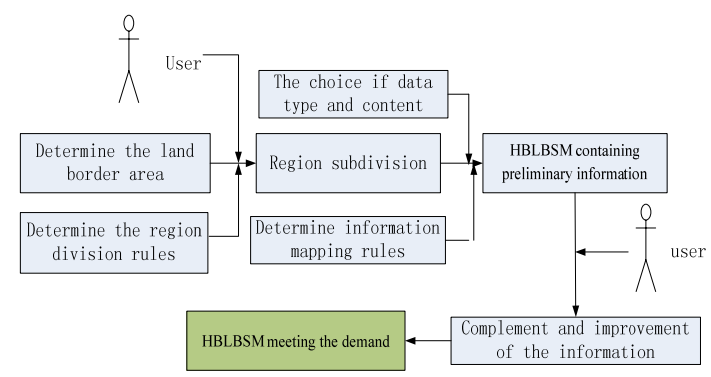

Figure 1. The flow chart of the model construction

\section{THE KEY TECHNOLOGIES OF THE MODEL}

In the process of building model, the technologies of how to determine to the mathematical basis, how to choose the region subdivision method, how to constitute the information mapping rules and so on all play a key role. The following is described respectively.

\subsection{Determination of Mathematical Foundation}

Compared with the global region, the land boundary region is small. For the sake of simplicity, we take the land boundary region as the plane. In addition, in order to meet the needs of modeling better, we choose the Conformal Conic Projection which the projection deformation is smaller[6][9].

In addition, in the process of land boundary analysis, there is a large number of position calculations. If the map projection coordinates are used in each location calculation, it will be Cumbersome and not intuitive. Therefore, in the subdivision process we choose the Cartesian coordinates to calculate, and when the calculation and analysis is completed, convert it to the original coordinate system through the method of coordinate transformation. Suppose there is a land border area A, we took the lower left corner point of the minimum area rectangle as the origin of coordinates, upward as the $\mathrm{x}$ axis, the right as the $\mathrm{Y}$-axis, and establish Cartesian coordinate system. The results is shown in figure 2 .

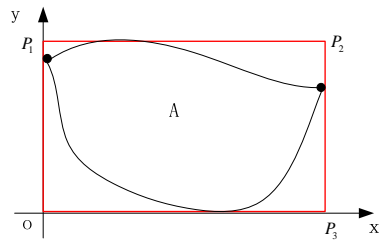

Figure 2. Schematic diagram of local Cartesian coordinates

If the coordinate of the rectangular coordinate system origin $O_{\text {is }}\left(\mathrm{x}_{W}^{\prime}, \mathrm{y}_{S}^{\prime}\right)$ in the geodetic coordinate system, the top right corner point $\mathrm{P}_{2}$ is $\left(\mathrm{x}_{E}^{\prime}, \mathrm{y}_{N}^{\prime}\right)$, then the coordinate position of any point $\mathrm{q}(\mathrm{x}, \mathrm{y})$ in the rectangular coordinate system is as follows in the geodetic coordinate system:

$$
\left\{\begin{array}{l}
x^{\prime}=x_{W}^{\prime}+x \\
y^{\prime}=y_{S}^{\prime}+y
\end{array}\right.
$$


Conversion from coordinates to geodetic coordinates to Cartesian coordinates is similarly.

\subsection{The Choice of Region Subdivision Method}

When the disputed area is determined, formulating regional subdivision rules to split region is the first problem to construct the HBLBSM. Here, the author analyzes the various regional subdivision method, summarizes its weakness applied in the field of land boundary demarcation, and on the basis of this puts forward the subdivision method suitable for the land border demarcation. It includes the first division and level division two processes.

\subsubsection{First Division to The Region}

The subdivision to the land border area for the first time mainly includes the following steps:

(1) Determine the scope of the area

We should firstly clear the scope of the study area, namely the subdivision object. We can see the land border area as a flat area, the size of the area is regional minimum circumscribed rectangle $\mathrm{OP}_{1} \mathrm{P}_{2} \mathrm{P}_{3}$. Four boundary point coordinates under the geodetic coordinate system are: $\mathrm{O}\left(\mathrm{x}_{W}^{\prime}, \mathrm{y}_{S}^{\prime}\right), P_{1}\left(\mathrm{x}_{W}^{\prime}, \mathrm{y}_{N}^{\prime}\right), P_{2}\left(\mathrm{x}_{E}^{\prime}, \mathrm{y}_{N}^{\prime}\right), P_{3}\left(\mathrm{x}_{E}^{\prime}, \mathrm{y}_{S}^{\prime}\right)$, the length and width of the area are: Length $=\mathrm{x}_{E}^{\prime}-\mathrm{x}_{W}^{\prime}$, Width $=\mathrm{y}_{N-}^{\prime} \mathrm{y}_{S}^{\prime}$. (2) Determine the digital map scale

Determining the digital map scale within the rectangular region, namely: the unit area of the map to the actual distance length. We assume it is $\mathrm{d}$, the unit is kilometers.

\section{(3) Determine the subdivision density}

The subdivision density, also called the subdivision grid resolution, is decided by the hexagon grid size. In the same region, when the hexagon is larger, the subdivision density is small, the resolution is smaller. Hexagon size is expressed by the length of hexagon lattice, also its circumcircle radius $\mathrm{R}$. The unit of $\mathrm{R}$ is kilometer. Its size in the Cartesian coordinate system is $r=R / d$.

(4) Determine the Subdivision starting point

As shown in the figure below, assuming that the starting point of subdivision for the rectangular coordinate system origin, the coordinate of the first hexagon center is $(0,0)$.

\section{(5) Begin to split}

After Determining the starting subdivision point and the first hexagon center point, the regional subdivision shape has been basically determined. The following figure is the regional subdivision overview:

We temporarily to the center of each hexagon to represent the whole hexagon, the calculation process is as follows: Set:

$$
t_{1}=\frac{\text { Length }}{1.5 * r}, t_{2}=\frac{\text { Width }}{\sqrt{3} * r}
$$

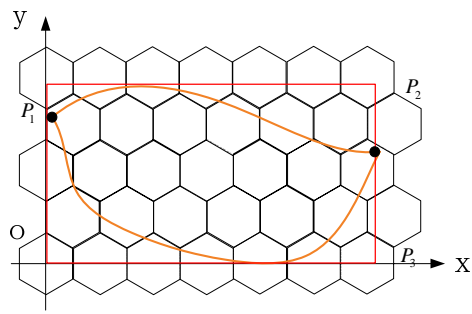

Figure 4. Regional subdivision overview diagram Then:

Among them:

$$
\begin{aligned}
& y_{i}=1.5 * r *(i-1),(i=1, \cdots, n) \\
& x_{j}=\left\{\begin{array}{c}
\frac{\sqrt{3} * r}{2} *(2 * j-1), i=2,4,6, \cdots \\
\sqrt{3} * r *(j-1), i=1,3,5, \cdots
\end{array}\right. \\
& (j=1,2, \cdots, m)
\end{aligned}
$$

$$
\begin{aligned}
& n=\left\{\begin{array}{l}
\bmod \left(t_{1}\right)+1, \bmod \left(t_{1}\right)=t_{1} \\
\bmod \left(t_{1}\right)+2, \bmod \left(t_{1}\right) \neq t_{1}
\end{array}\right. \\
& m=\left\{\begin{array}{l}
\bmod \left(t_{2}\right)+1, \bmod \left(t_{2}\right)=t_{2} \\
\bmod \left(t_{2}\right)+2, \bmod \left(t_{2}\right) \neq t_{2}
\end{array}\right.
\end{aligned}
$$

$\mathrm{I}$ is the hexagon row number, $\mathrm{j}$ is the hexagon column number.

\subsubsection{Level Division to The Region}

On the basis of the above subdivision segmentation process includes the following several ways:

(1) The first subdivision

The first subdivision to the area is a reference to the PYXIS hierarchy method. Specifically, the hexagon in the odd numbered rows is subdivided into one hexagon, the hexagon in the even numbered rows is subdivided into five hexagons, the right most hexagons is subdivided into seven hexagons. As shown below:

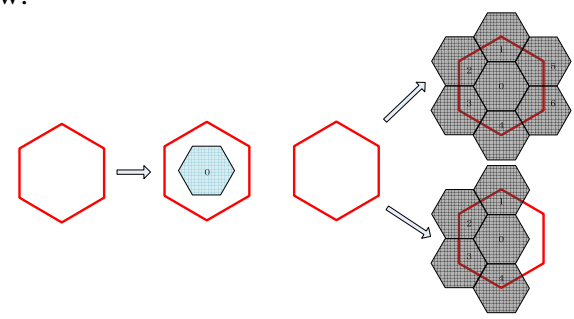

Figure 5. The first subdivision process diagram

(2) The nth subdivision

Subdivision method after second times are similar. They comply with the following principles: If the center of the hexagon is the same with the center of the last layer hexagon, the hexagon can be subdivided into seven hexagons; if the center of the hexagon is the same with the vertex of the last layer hexagon, the hexagon can be subdivided into one hexagon. This subdivision method can ensure that the generated hexagonal grid subordinate relations are very clear, that is the hexagons next layer are generated by one hexagon last layer. It is good for our traversal and analysis of the grid.

\subsection{Information Mapping Rules}

Completing the land boundary region partition just complete the regional geographic subdivision. At this time each hexagon has only geography information, without any other 


\section{5 - 6 December 2013, Beijing, China}

information. Information mapping is designed to make the statistical thematic information, terrain feature information, indivisible information and other information in the region mapping to the edges and units of each hexagon, which lay the foundation for all kinds of information in decision making.

\subsubsection{Statistical Thematic Information Mapping}

Statistical thematic information refers to the information that outstanding represent the natural or social economic phenomenon and the relevant characteristics, usually includes average information and total information. Their corresponding mapping method is improved dominated area method.

The total information, namely the corresponding attribute value is the value that overall properties of the area, such as total population. For this type of information processing is: firstly, calculate the average value according to the area, and then Multiply their area with the average value, finally put the values obtained are the attribute value of the grid. For example, there are three different attribute domain in the hexagon and the property be mapping for is the total population. Is first of all, we calculate the average value according to the area. After the calculation, the value of A face is 40 , the value of $B$ face is 50 and the value of $\mathrm{C}$ face is 30 . The three surface area in the hexagon were $10,20,20$. Then the total population of hexagon is as follows:

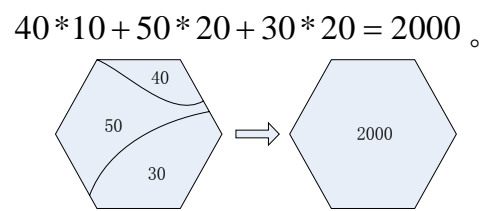

Figure 6. The total information mapping diagram

The average information, namely the corresponding attribute value is the value that average properties of the area, such as population density. For this type of information processing is: calculate the average value according to the weighted area, as the value of the grid property. For example, there are three different attribute domain in the hexagon and the property be mapping for is the population density. After the calculation, the value of $A$ face is 40 , the value of $B$ face is 50 and the value of $\mathrm{C}$ face is 30 . The three surface area in the hexagon were $10,20,20$. Then the population density of hexagon is as follows:

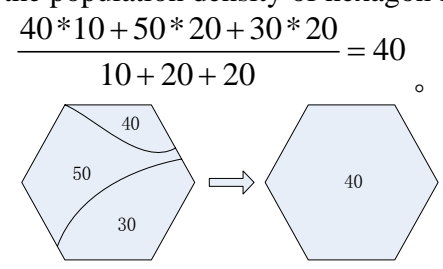

Figure 7. The average information mapping diagram

To be sure, after mapping these attributes information corresponds to the attribute values of the hexagon units. The following two types of information mainly corresponds to the attribute values of the hexagon units and their edges after mapping.

\subsubsection{Indivisible Information Mapping}

In the land boundary information, some descriptive or qualitative information such as: religious, cultural, political, traditional, etc., have a great influence on the delimitation and analysis and decision, but it cannot be quantified, and therefore cannot be involved in the model building. Aimed at this situation, the author analysis and found these information content, while large difference, but there is a common manifestation: the integral whole. For this kind of information, in addition to mapping the qualitative and quantitative properties information itself, but also mapping the properties affecting. Therefore, the author put the impact results of these information mapping to the attributes of relevant hexagons and the connecting lines between them. Specifically, we set the dividing attribute value of the related hexagon units to 0 , said indivisible; the connected attribute value of the related connecting lines between hexagons to 0 , said disconnected.

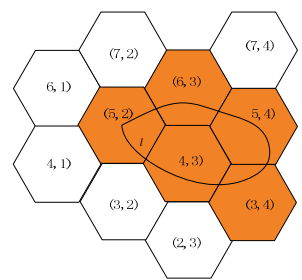

Figure 8. Indivisible area mapping results in the model

As shown in figure 8, the area curve 1 enveloping is an integral area. We put the integral information mapping to the model of the region, its corresponding hexagon units respectively are the dark hexagon units $(5,2),(4,3),(6,3),(5,4),(3,4)$. The dividing attribute values of these hexagon units are 0 , and the connected attribute values of the related connecting lines between these hexagons are also 0 .

\subsubsection{Terrain Feature Information Mapping}

Terrain feature information is mainly refers to the point, line, or planar elements which have control function in the spatial distribution of the surface topography. Terrain feature elements constitutes the basic framework of surface topography and rolling.

For the mapping of this kind of information, we have to consider from two aspects. Firstly, we should ensure the integrity of the feature information. Secondly, we must be able to make these information directly involved in the modeling and analysis. For this, we set up division properties for each hexagon, cross properties for each edge of the hexagons. In normal conditions, the division property value and the cross property value are the default value. When the characteristic line through some hexagons, the properties of the corresponding units and their edges will be changed. The division property value of the corresponding unit will be changed to 1 , division first; the cross property of the edges will be changed to 1 , through first.

For example, a ridge line ab cross through four hexagons $(3,2)$, $(3,3),(4),(4,5)$. On the one hand, the division property values of the four hexagons have changed to 1 . On the other hand, the cross property values of the edges that the ridge line intersect are also changed to 1 .

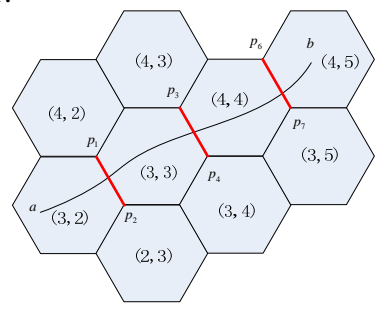

Figure 9. Terrain feature information mapping results 


\section{THE EXPERIMENT AND SUMMARY}

According to the above model building method, we can implement the subdivision and mapping of any disputed area. If there is a disputed area A, we can construct the HBLBSM using the above method. In three layer subdivision structure, for example, the level subdivision process of the region $\mathrm{A}$ as shown in figure 10. Because the mapping information is complicated, the mapping results is no longer show.

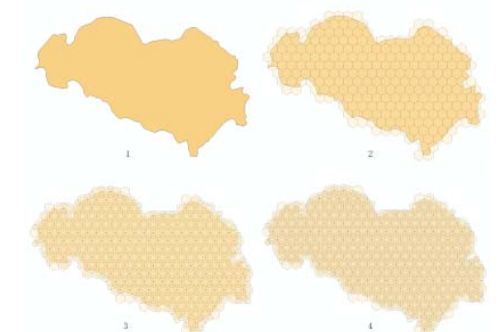

Figure 10. The model construction results

In conclusion, the author puts forward the Hexagon-Based Land Boundary Surroundings Model(HBLBSM) base on the analysis of the land border environment information. This paper discusses the key technologies of model building, including determination of mathematical foundation, the choice of region subdivision method, Information mapping method and so on. The building of the model can better support the space position analysis and attribute correlation analysis, laid a foundation for generating the land border demarcation.

\section{REFERENCES}

[1] The national boundary problems - colonial rule brought about by the China Education and research network.

[2] The sovereign territory disputes in the world list - Wikipedia. [3] Wu Lili. Research on the Auxiliary Generation of Initial Delimitation Line Based on Hexagon [D]. PLA Information Engineering University for the Degree of Doctor of Engineering, 2013

[4] Wu Lili, Hua Yixin, Xu Qing. The Study on the Land boundary environment model based on the hexagon [J]. Mappingand geospatial information, 2013 (2): 22-26.

[5] Cao Yibing. Research on the land border demarcation technology [D]. PLA Information Engineering University for the Degree of Master of Engineering, 2011

[6] Liu Hehui. Land boundary demarcation negotiation decision support system and its application[D]. Zhengzhou: PLA Information Engineering University for the Degree of Doctor of Engineering, 2011

[7] Zhang Wen. On the ancient Chinese view of the state historical coordinate and world view -the border and boundary formation[J]. Research on Chinese frontier history, 2007 (3): 16-23.

[8] Wang Hongwei. The rift border where [J]. Ethnic today, 2006 (8): 47-48.

[9] Peng Rencan. Maritime delimitation GIS technology research and practice [D]. Zhengzhou: PLA Information Engineering University for the Degree of Doctor of Engineering, 2003. 